\title{
The CONSORT statement: revised recommendations for improving the quality of reports of parallel-group randomised trials
}

\begin{abstract}
David Moher, Kenneth F Schulz, Douglas G Altman, for the CONSORT Group*
To comprehend the results of a randomised controlled trial (RCT), readers must understand its design, conduct, analysis, and interpretation. That goal can be achieved only through total transparency from authors. Despite several decades of educational efforts, the reporting of RCTs needs improvement. Investigators and editors developed the original CONSORT (Consolidated Standards of Reporting Trials) statement to help authors improve reporting by use of a checklist and flow diagram. The revised CONSORT statement presented here incorporates new evidence and addresses some criticisms of the original statement. The checklist items pertain to the content of the Title, Abstract, Introduction, Methods, Results, and Discussion. The revised checklist includes 22 items selected because empirical evidence indicates that not reporting this information is associated with biased estimates of treatment effect, or because the information is essential to judge the reliability or relevance of the findings. We intended the flow diagram to depict the passage of participants through an RCT. The revised flow diagram depicts information from four stages of a trial (enrolment, intervention allocation, followup, and analysis). The diagram explicitly shows the number of participants, for each intervention group, included in the primary data analysis. Inclusion of these numbers allows the reader to judge whether the authors have done an intentionto-treat analysis. In sum, the CONSORT statement is intended to improve the reporting of an RCT, enabling readers to understand a trial's conduct and to assess the validity of its results.
\end{abstract}

A report of a randomised controlled trial (RCT) should convey to the reader, in a transparent manner, why the study was undertaken, and how it was conducted and analysed. Inadequately reported randomisation, for example, has been associated with bias in estimating the effectiveness of interventions. ${ }^{1,2}$ To assess the strengths and limitations of an RCT, readers need and deserve to know the quality of its methods.

Despite several decades of educational efforts, RCTs are still not being reported adequately. ${ }^{3-6}$ For example, a review $^{5}$ of 122 recently published RCTs that assessed the effectiveness of selective serotonin reuptake inhibitors as a first-line management strategy for depression found that only one paper described randomisation adequately. Inadequate reporting makes the interpretation of RCTs difficult, if not impossible. Moreover, inadequate reporting borders on unethical practice when biased results receive false credibility.

\section{History of CONSORT}

In the mid-1990s, two independent initiatives to improve the quality of reports of RCTs led to the publication of the CONSORT statement, ${ }^{7}$ which was developed by an

Lancet 2001; 357: 1191-94

* Members listed at end of paper

The revised CONSORT statement is also published in JAMA 2001; 285: 1987-91 and Ann Intern Med 2001; 134: 657-62.

University of Ottawa, Thomas $\mathbf{C}$ Chalmers Centre for Systematic Reviews, Ottawa, Ontario, Canada (D Moher MSc); Family Health International and Department of Obstetrics and Gynecology, School of Medicine, University of North Carolina at Chapel Hill, NC, USA (K F Schulz PhD); and ICRF Medical Statistics Group and Centre for Statistics in Medicine, Institute of Health Sciences, Oxford, UK (D G Altman DSc)

Correspondence to: Dr Leah Lepage, Thomas C Chalmers Centre for Systematic Reviews, Children's Hospital of Eastern Ontario Research Institute, Room R235, 401 Smyth Road, Ottawa, Ontario K1H 8L1, Canada

(e-mail: Ilepage@uottawa.ca) international group of clinical trialists, statisticians, epidemiologists, and biomedical editors. CONSORT has been supported by a growing number of medical and health-care journals ${ }^{8-11}$ and editorial groups, including the International Committee of Medical Journal Editors (ICMJE, The Vancouver Group), ${ }^{12}$ the Council of Science Editors (CSE), and the World Association of Medical Editors (WAME). CONSORT is published in Dutch, English, French, German, Japanese, and Spanish. It can be accessed together with other information about the CONSORT group on the internet. ${ }^{13}$

The CONSORT statement consists of a checklist and flow diagram for reporting an RCT. For convenience, the checklist and diagram together are called simply CONSORT. They are primarily intended for use in writing, reviewing, or assessing reports of simple two-group parallel RCTs.

Preliminary data indicate that the use of CONSORT does indeed help to improve the quality of reports of RCTs. ${ }^{14,15}$ In an assessment ${ }^{14}$ of 71 RCTs, published in three journals in 1994, allocation concealment was not clearly reported in $43(61 \%)$ of the trials. 4 years later, after these three journals required that authors reporting an RCT use CONSORT, the proportion of papers in which allocation concealment was not clearly reported had dropped to 30 of 77 (39\%, mean difference $-22 \%$ [95\% CI -38 to -6$])$.

The usefulness of CONSORT is increased by continuous monitoring of biomedical publications, which allows it to be modified dependent on the merits of maintaining or dropping current items, and including new items. For example, when Meinert ${ }^{16}$ observed that the flow diagram did not provide important information about the number of participants who entered each phase of an RCT (ie, enrolment, treatment allocation, follow-up, and data analysis), the diagram could be modified to accommodate the information. The checklist is similarly flexible.

This iterative process makes the CONSORT statement a continually evolving instrument. Although participants in the CONSORT group and their degree of involvement vary over time, members meet regularly to review the need to 
refine CONSORT. At the 1999 meeting, the participants decided to revise the original statement. This report reflects changes determined by consensus of the CONSORT group, partly in response to emerging evidence on the importance of various elements of RCTs.

\section{Revision of the CONSORT statement}

13 members of the CONSORT group met in May, 1999, with the main objective of revising the original CONSORT checklist and flow diagram, as needed. The group discussed the merits of including each item in the light of current evidence. As in developing the original CONSORT statement, our intention was to keep only those items deemed fundamental to reporting standards for an RCT. Some items not regarded as essential could well be highly desirable and should still be included in an RCT report even though they are not included in CONSORT. Such items include approval of an institutional ethics review board, sources of funding for the trial, and a trial registry number-eg, the International Standard Randomized Controlled Trial Number (ISRCTN) used to register the RCT at its inception. ${ }^{17}$

Shortly after the meeting, a revised version of the checklist was circulated to the group for additional comments and feedback. Revisions to the flow diagram were similarly made. All these changes were discussed when CONSORT participants met in May, 2000, and the revised statement was finalised shortly afterwards.

The revised CONSORT statement includes a 22 -item checklist (table) and a flow diagram (figure). Its main aim is to help authors improve the quality of reports of simple two-group parallel RCTs. However, the basic philosophy underlying the development of the statement can be applied to any design. In this respect, additional statements for other designs will be forthcoming from the group. CONSORT can also be used by peer reviewers and editors to identify reports with inadequate description of trials and those with potentially biased results. ${ }^{1,2}$

During the 1999 meeting, the group also discussed the benefits of developing an explanatory document to improve the use and dissemination of CONSORT. The document is patterned on reporting of statistical aspects of clinical research, ${ }^{18}$ and was developed to help facilitate the recommendations of the ICMJE's Uniform Requirements for Manuscripts Submitted to Biomedical Journals. Three members of the CONSORT group, with assistance from members on some checklist items, drafted an explanation and elaboration document. That document ${ }^{19}$ was circulated to the group for additions and revisions and was last revised after review at the latest CONSORT group meeting.

\section{Changes to CONSORT}

(1) In the revised checklist, a new column for "Paper section and topic" integrates information from the "Subheading" column that was contained in the original statement.

(2) The "Was it reported?" column has been integrated into a "reported on page number" column, as requested by some journals.

(3) Each item of the checklist is now numbered and the syntax and order have been revised to improve the flow of information.

(4) "Title" and "Abstract" are now combined in the first item.

(5) Although the content of the revised checklist is similar to the original, some items that were previously combined

\begin{tabular}{|c|c|c|c|}
\hline & $\begin{array}{l}\text { Item } \\
\text { number }\end{array}$ & Descriptor & $\begin{array}{l}\text { Reported on } \\
\text { page number }\end{array}$ \\
\hline Title and abstract & 1 & How participants were allocated to interventions (eg, "random allocation", "randomised", or "randomly assigned"). & \\
\hline \multicolumn{4}{|l|}{ Introduction } \\
\hline Background & 2 & Scientified background and explanation of rationale. & \\
\hline \multicolumn{4}{|l|}{ Methods } \\
\hline Participants & 3 & Eligibility criteria for participants and the settings and locations where the data were collected. & \\
\hline Interventions & 4 & Precise details of the interventions intended for each group and how and when they were actually administered. & \\
\hline Objectives & 5 & Specific objectives and hypotheses. & \\
\hline Outcomes & 6 & $\begin{array}{l}\text { Clearly defined primary and secondary outcome measures and, when applicable, any methods used to enhance the quality of } \\
\text { measurements (eg, multiple observations, training of assessors, \&c). }\end{array}$ & \\
\hline Sample size & 7 & How sample size was determined and, when applicable, explanation of any interim analyses and stopping rules. & \\
\hline \multicolumn{4}{|l|}{ Randomisation } \\
\hline Sequence generation & 8 & Method used to generate the random allocation sequence, including details of any restriction (eg, blocking, stratification). & \\
\hline Allocation concealment & 9 & $\begin{array}{l}\text { Method used to implement the random allocation sequence (eg, numbered containers or central telephone), clarifying } \\
\text { whether the sequence was concealed until interventions were assigned. }\end{array}$ & \\
\hline Implementation & 10 & Who generated the allocation sequence, who enrolled participants, and who assigned participants to their groups. & \\
\hline Blinding (masking) & 11 & $\begin{array}{l}\text { Whether or not participants, those administering the interventions, and those assessing the outcomes were aware of group } \\
\text { assignment. If not, how the success of masking was assessed. }\end{array}$ & \\
\hline Statistical methods & 12 & $\begin{array}{l}\text { Statistical methods used to compare groups for primary outcome(s); methods for additional analyses, such as subgroup } \\
\text { analyses and adjusted analyses. }\end{array}$ & \\
\hline \multicolumn{4}{|l|}{ Results } \\
\hline Participant flow & 13 & $\begin{array}{l}\text { Flow of participants through each stage (a diagram is strongly recommended). Specifically, for each group, report the numbers } \\
\text { of participants randomly assigned, receiving intended treatment, completing the study protocol, and analysed for the primary } \\
\text { outcome. Describe protocol deviations from study as planned, together with reasons. }\end{array}$ & \\
\hline Recruitment & 14 & Dates defining the periods of recruitment and follow-up. & \\
\hline Baseline data & 15 & Baseline demographic and clinical characteristics of each group. & \\
\hline Numbers analysed & 16 & $\begin{array}{l}\text { Number of participants (denominator) in each group included in each analysis and whether the analysis was by "intention to } \\
\text { treat". State the results in absolute numbers when feasible (eg, 10/20, not } 50 \% \text { ). }\end{array}$ & \\
\hline Outcomes and estimation & 17 & $\begin{array}{l}\text { For each primary and secondary outcome, a summary of results for each group, and the estimated effect size and its } \\
\text { precision }(\mathrm{eg}, 95 \% \mathrm{Cl}) \text {. }\end{array}$ & \\
\hline Ancillary analyses & 18 & $\begin{array}{l}\text { Address multiplicity by reporting any other analyses performed, including subgroup analyses and adjusted analyses, } \\
\text { indicating those prespecified and those exploratory. }\end{array}$ & \\
\hline Adverse events & 19 & All important adverse events or side-effects in each intervention group. & \\
\hline \multicolumn{4}{|l|}{ Discussion } \\
\hline Interpretation & 20 & $\begin{array}{l}\text { Interpretation of the results, taking into account study hypotheses, sources of potential bias or imprecision and the dangers } \\
\text { associated with multiplicity of analyses and outcomes. }\end{array}$ & \\
\hline Generalisability & 21 & Generalisability (external validity) of the trial findings. & \\
\hline Overall evidence & 22 & General interpretation of the results in the context of current evidence. & \\
\hline
\end{tabular}

Checklist of items to include when reporting a randomised trial 
are now separate. For example, previously authors were asked to describe "primary and secondary outcome(s) measure(s) and the minimum important difference(s), and indicate how the target sample size was projected". In the new version, issues pertaining to outcomes (item 6) and sample size (item 7) are separate, enabling authors to be more explicit about each. Moreover, some items request additional information. For example, for outcomes, authors are asked to report any methods used to improve the quality of measurements, such as multiple observations.

(6) The item asking for the unit of randomisation (eg, cluster) has been dropped because specific checklists have been developed for reporting cluster $\mathrm{RCTs}^{20}$ and other design type ${ }^{13}$ since publication of the original checklist.

(7) Whenever possible, new evidence is incorporated into the revised checklist. For example, authors are asked to be explicit about whether the analysis reported is by intention to treat (item 16). This request is based partly on the observations ${ }^{21}$ that authors do not adequately describe and apply intention-to-treat analysis, and that reports not providing this information are less likely to provide other relevant information such as losses to follow-up. ${ }^{22}$

(8)The revised flow diagram depicts information from four stages of a trial (enrolment, intervention allocation, followup, and analysis). The revised diagram explicitly shows the number of participants, for each intervention group, included in the primary data analysis. Inclusion of these numbers lets the reader know whether the authors have done an intention-to-treat analysis. ${ }^{21-23}$ Because some of the information might not always be known, and to accommodate other information, the structure of the flow diagram might need to be modified for a particular trial. Inclusion of the participant flow diagram in the report is strongly recommended but might be unnecessary for simple trials such as those without any participant withdrawals or dropouts.

\section{Discussion}

Specifically developed to guide authors about how to improve the quality of reporting of simple two-group parallel RCTs, CONSORT encourages transparency with reporting of the methods and results so that reports of RCTs can be interpreted readily and accurately. However, CONSORT does not address other facets of reporting that also require attention, such as scientific content and readability of RCT reports. Some authors in their enthusiasm to use CONSORT have modified the checklist. ${ }^{24}$ We recommend against such modifications because they could be based on a different process from the one used by the CONSORT group.

The use of CONSORT seems to reduce, if not eliminate, inadequate reporting of RCTs. ${ }^{14,15}$ Potentially, the use of CONSORT should positively influence the manner in which RCTs are conducted. Granting agencies have noted this potential relation, and in at least one case ${ }^{25}$ have encouraged researchers to consider in their application how they have dealt with the CONSORT items.

The evidence-based approach used to develop CONSORT has also been used to develop standards for reporting meta-analyses of randomised trials, ${ }^{26}$ metaanalyses of observational studies, ${ }^{27}$ and diagnostic studies (Jeroen Lijmer, personal communication). Health economists have also started to develop reporting standards ${ }^{28}$ to help to improve the quality of their reports. ${ }^{29}$ The intent of all these initiatives is to improve the quality of reporting of biomedical research, ${ }^{30}$ and by doing so, to bring about more effective health care.

The revised CONSORT statement will replace the original one in the journals and groups that already support

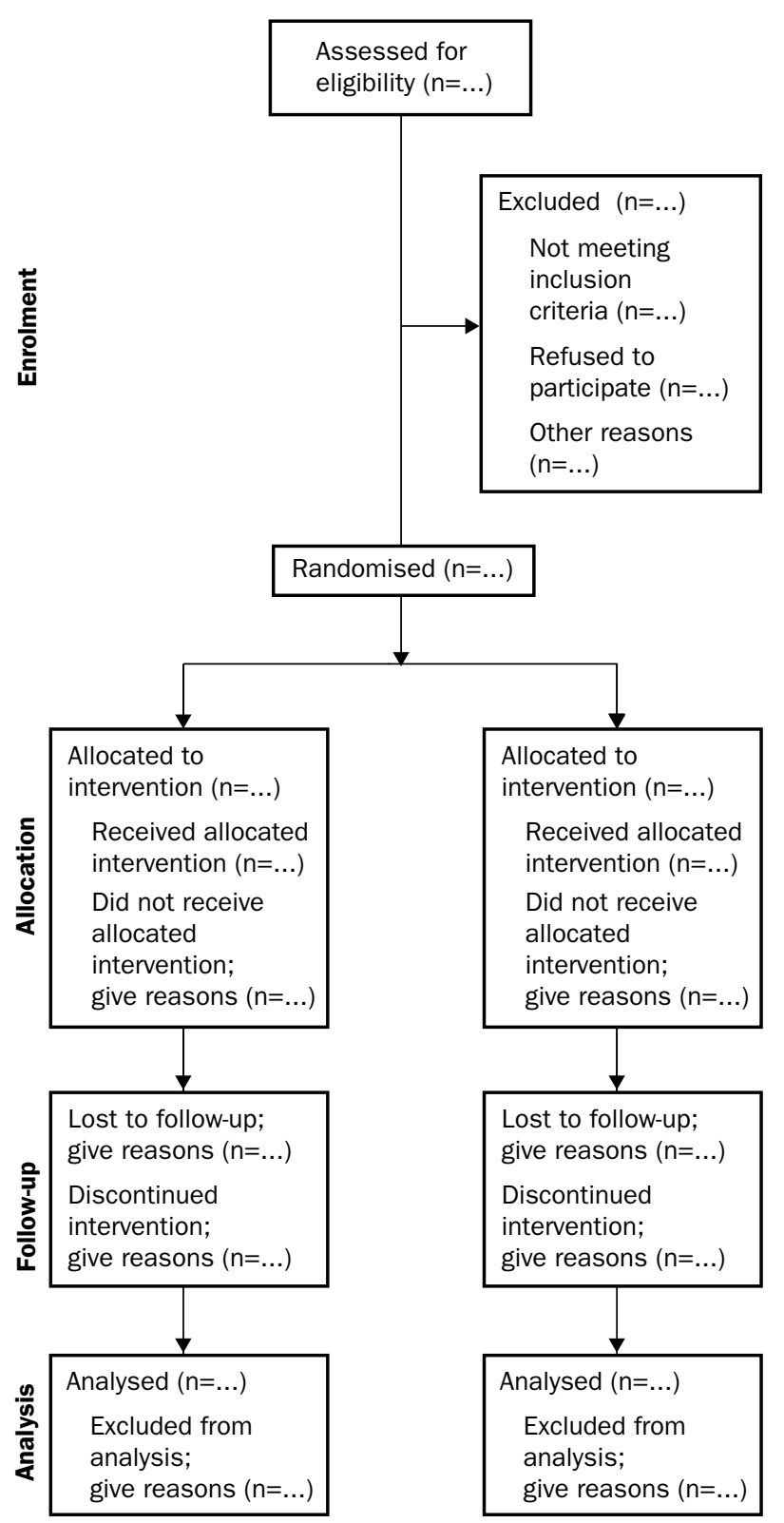

Flow diagram of the progress through the phases of a randomised trial

it. Journals that do not yet support CONSORT may do so by registering on the CONSORT website. ${ }^{13}$ To convey to authors the importance of improved quality in the reporting of RCTs, we encourage supporting journals to reference the revised CONSORT statement and the CONSORT internet address in their Instructions to Contributors. Since the journals publishing the revised CONSORT statement have waived copyright protection, CONSORT is now widely accessible to the biomedical community. The CONSORT checklist and flow diagram can also be accessed at the CONSORT website.

A lack of clarification of the meaning and rationale for each checklist item in the original CONSORT statement has been remedied with the development of the CONSORT explanation and elaboration document, ${ }^{19}$ which can also be found on the CONSORT website. This document reports the evidence on which the checklist items are based, including the references, which had annotated the checklist items in the previous version. We also 
encourage journals to include reference to this document in their Instructions to Contributors.

Emphasising the evolving nature of CONSORT, the CONSORT group invites readers to comment on the updated checklist and flow diagram through the CONSORT website. ${ }^{13}$ Comments and suggestions will be collated and considered at the next meeting of the group in 2001.

\section{Contributors}

David Moher, Ken Schulz, and Doug Altman participated in regular conference calls, identified participants, participated in the CONSORT meetings, and drafted the paper. David Moher and Leah Lepage planned the CONSORT meetings, identified and secured funding, invited the participants, and planned the meeting agenda. The members of the CONSORT group listed below attended the CONSORT meetings and provided input towards the revised checklist, flow diagram, and text.

\section{The CONSORT group}

Frank Davidoff (Annals of Internal Medicine, Philadelphia, PA, USA); Susan Eastwood (University of California at San Francisco, CA, USA); Matthias Egger (Department of Social Medicine, University of Bristol, UK); Diana Elbourne (London School of Hygiene and Tropical Medicine, London, UK); Peter Gøtzsche (Nordic Cochrane Centre, Copenhagen, Denmark); Sylvan B Green (School of Medicine, Case Western Reserve University, Cleveland, OH, USA); Leni Grossman (Merck \& Co, Whitehouse Station, NJ, USA); Barbara S Hawkins (Wilmer Ophthalmological Institute, Johns Hopkins University, (Baltimore, MD, USA); Richard Horton (The Lancet, London, UK); Wayne B Jonas (Uniformed Services University of the Health Sciences, Bethesda, MD); Terry Klassen (Department of Pediatrics, University of Alberta, Edmonton, Alberta, Canada); Leah Lepage (Thomas C Chalmers Centre for Systematic Reviews, Ottawa, Ontario, Canada); Thomas Lang (Tom Lang Communications, Lakewood, OH, USA); Jeroen Lijmer (Department of Clinical Epidemiology, University of Amsterdam, Netherlands); Rick Malone (TAP Pharmaceuticals, Lake Forest, IL, USA); Curtis L Meinert (Johns Hopkins University, Baltimore, MD); Mary Mosley (Life Science Publishing, Tokyo, Japan); Stuart Pocock (London School of Hygiene and Tropical Medicine, London); Drummond Rennie (Fournal of the American Medical Association, Chicago, IL); David S Riley (University of New Mexico Medical School, Santa Fe, NM, USA); Roberta W Scherer (Epidemiology \& Preventive Medicine, University of Maryland School of Medicine, Baltimore, MD); Ida Sim (University of California at San Francisco, CA); Donna Stroup (Epidemiology Program Office, Centers for Disease Control and Prevention, Atlanta, GA, USA).

\section{Acknowledgments}

The effort to improve the reporting of randomised trials, from its beginning with the Standards of Reporting Trials (SORT) group to the current activities of the Consolidated Standards of Reporting Trials (CONSORT) group, has involved many people around the globe. We thank Leah Lepage for keeping everybody all lined up and moving in the same direction.

Financial support to convene meetings of the CONSORT group was provided in part by Abbott Laboratories, American College of Physicians, GlaxoWellcome, The Lancet, Merck, the Canadian Institutes for Health Research, National Library of Medicine, and TAP Pharmaceuticals.

\section{References}

1 Schulz KF, Chalmers I, Hayes RJ, Altman DG. Empirical evidence of bias: dimensions of methodological quality associated with estimates of treatment effects in controlled trials. FAMA 1995; 273: 408-12.

2 Moher D, Pham B, Jones A, et al. Does the quality of reports of randomised trials affect estimates of intervention efficacy reported in meta-analyses? Lancet 1998; 352: 609-13.

3 Jadad AR, Boyle M, Cunningham C, Kim M, Schachar R. Treatment of attention deficit/hyperactivity disorder: evidence report/technology assessment no 11. Hamilton: McMaster University, 2000.
4 Thornley B, Adams CE. Content and quality of 2000 controlled trials in schizophrenia over 50 years. BMF 1998; 317: 1181-84.

5 Hotopf M, Lewis G, Normand C. Putting trials on trial- the costs and consequences of small trials in depression: a systematic review of methodology. F Epidemiol Community Health 1997; 51: 354-58.

6 Dickinson K, Bunn F, Wentz R, Edwards P, Roberts I. Size and quality of randomised controlled trials in head injury: review of published studies. BMF 2000; 320: 1308-11.

7 Begg CB, Cho MK, Eastwood S, et al. Improving the quality of reporting of randomized controlled trials: the CONSORT statement. FAMA 1996; 276: 637-39.

8 Freemantle N, Mason JM, Haines A, Eccles MP. CONSORT: an important step toward evidence-based health care. Ann Intern Med 1997; 126: 81-83.

9 Altman DG. Better reporting of randomized controlled trials: the CONSORT statement. BMF 1996; 313: 570-71.

10 Schulz KF. The quest for unbiased research: randomized clinical trials and the CONSORT reporting guidelines. Ann Neurol 1997; 41: 569-73.

11 Huston P. Hoey J. CMAf endorses the CONSORT statement. Can Med Assoc F 1996; 155: 1277-79.

12 Davidoff F. News from the International Committee of Medical Journal Editors. Ann Intern Med 2000; 133: 229-31.

13 www.consort-statement.org (accessed Feb 15, 2001).

14 Moher D, Jones A, Lepage L, for the CONSORT Group. Use of CONSORT statement and quality of reports of randomized trials: a comparative before and after evaluation? $\mathcal{F A M A}$ (in press).

15 Egger M, Juni P, Bartiett C, for the CONSORT Group. The value of patient flow charts in reports of randomized controlled trials: bibliographic study. $\mathscr{F} A M A$ (in press).

16 Meinert CL. Beyond CONSORT: need for improved reporting standards for clinical trials. $f A M A$ 1998; 279: 1487-89.

17 Chalmers I. Current Controlled Trials: an opportunity to help improve the quality of clinical research. Curr Control Trials Cardiovasc Med 2000; 1: 3-8.

18 Bailer JC III, Mosteller F. Guidelines for statistical reporting in articles for medical journals: amplifications and explanations. Ann Intern Med 1988; 108: 266-73.

19 Altman DG, Schulz KF, Moher D, et al, for the CONSORT group. The revised CONSORT statement for reporting randomized trials: explanation and elaboration. Ann Intern Med 2001; 134: 663-94.

20 Elbourne DR, Campbell MK. Extending the CONSORT statement to cluster randomised trials: for discussion. Stat Med (in press).

21 Hollis S, Campbell F. What is meant by intention-to-treat analysis? Survey of published randomized controlled trials. BMF 1999; 319: 670-74.

22 Ruiz-Canela M. Martinez-Gonzalez MA, de Irala-Estevez J. Intentionto-treat analysis is related to methodological quality. BMf 2000; 320: 1007.

23 Lee YJ, Ellenberg JH, Hirtz DG, Nelson KB. Analysis of clinical trials by treatment actually received: is it really an option? Stat Med 1991; 10: 1595-605.

24 Bentzen SM. Towards evidence based radiation oncology: improving the design, analysis, and reporting of clinical outcome studies in radiotherapy. Radiother Oncol 1998; 46: 5-18.

25 O'Toole LB. MRC uses checklist similar to CONSORT's. BMF 1997; 314: 1127.

26 Moher D, Cook DJ, Eastwood S, Olkin I, Rennie D, Stroup DF, for the QUOROM group. Improving the quality of reports of meta-analyses of randomised controlled trials: the QUOROM statement. Lancet 1999; 354: 1896-900.

27 Stroup DF, Berlin IA, Morton SC, et al. Meta-analysis of observational studies in epidemiology: a proposal for reporting. $\mathscr{F} A M A 2000 ; \mathbf{2 8 3}$ : 2008-12.

28 Siegel IE, Weinstein MC, Russell LB, Gold MR. Recommendations for reporting cost-effectiveness analysis. $\mathcal{F} A M A$ 1996; 276: 1339-41.

29 Neumann PJ, Stone PW, Chapman RH, Sandberg EA, Bell CM. The quality of reporting in published cost-utility analyses, 1976-1997. Ann Intern Med 2000; 132: 964-72.

30 Altman DG. The scandal of poor medical research. BMF 1994; 308: 283-84 